\title{
Strategies for pro-active case-finding in leprosy control
}

\author{
P. R. KLATSER \\ Department of Biomedical Research, Royal Tropical Institute, \\ Amsterdam, The Netherlands
}

For many years, the control of leprosy has been based on the passive and sometimes active detection of patients, followed by treatment with multidrug therapy (MDT). This has resulted in a decline of prevalence, but there is little evidence that incidence is declining. In some areas, leprosy-control is or soon will be integrated into the general health services; in other areas 'vertical' programmes of leprosy-control will continue to exist. In either situation, the numbers of patients will be small. In this new, 'post-elimination' era, new approaches and strategies to eliminate leprosy as a public health problem in defined geographic areas are required. ${ }^{1}$

Recent insight into the epidemiology of leprosy has resulted in the recognition that leprosy is a very infectious disease, analogous to tuberculosis, but that few of the infected persons develop the disease. ${ }^{2}$ The risk of disease is inversely related to the degree of contact with an infectious patient. It has also been shown that the majority of new patients have been in contact with another, known patient. ${ }^{3}$ This has led to the 'stone-in-the-pond' model, similar to that developed for tuberculosis, as a description of the means by which transmission of Mycobacterium leprae occurs in concentric circles around a patient. This model offers tools for improved leprosy control, by redirecting control-activities from the current 'blanket' approach to a more focused approach, which includes intervention strategies applied to defined groups of contacts. ${ }^{3}$

Two questions arise. Why do not all patients transmit the infection? And can transmission be interrupted by prophylactic treatment of contacts? The first question is more scientific in nature, but may have practical implications as well. It is well known that some patients appear to be less effective in transmitting the organism than are other patients, whether MB or not. No secondary cases are to be found in the environment of the former group of patients, suggesting that transmission has stopped. In our studies in Indonesia, $47 \%$ of the MB patients and $63 \%$ of the PB patients did not generate new cases. ${ }^{3}$ This phenomenon may result from differing degrees of infectiousness of the patients, or from a lower degree of virulence of the strain of $M$. leprae involved, or from variations of susceptibility of those in contact with these patients, or from any combination of these three factors. Intervention in the chain of transmission may be targeted more effectively if both the degree of infectiousness of patients and the degree of susceptibility of contacts could be better defined. However, from an operational point of view, it will be more feasible to focus on the infectiousness of the patients.

The answer to the second question, concerning categories of contacts to be administered chemoprophylaxis in order to interrupt transmission of the organism, will help in formulating 
a policy for targeting high-risk groups for intervention as a leprosy control measure. From the perspectives of costs and operational feasibility, one would like to minimize the numbers of contacts to be prophylactically treated. Therefore, there is a need to determine which categories, and thereby the number, of contacts to be included in the intervention, in order to achieve interruption of the chain of infection.

We are currently studying a number of indicators of infectiousness, with the aim of finding effective tools for early detection and prevention of leprosy that can be applied in the field, in island populations in Indonesia. Island populations were chosen because such populations are relatively stable and allow good demarcation of the concentric circles of contact around a patient. On five small islands in South Sulawesi Province, Indonesia, 3987 ( $84 \%$ ) of a total population of 4770 persons were screened for leprosy in June and July 2000. A total of 91 new leprosy cases were diagnosed, representing a case-detection rate of $2.3 \%$, with a range of $1 \cdot 2-5 \cdot 0 \%$ per island. The prevalence rate was 191 per 10,000 , with a range of $87-442$ per 10,000 per island. Forty-six percent of the patients were classified MB, $40 \%$ single-lesion PB, and 14\% PB with two to five lesions. Children under 15 years of age represented $9 \%$ of the patients, and $11 \%$ of the patients demonstrated WHO grade 2 disability.

Sixty-eight (75\%) of the patients were clustered, where clustering is defined as a group of at least two patients who either fall in each other's contact group or share the same contacts. In this study, contacts are defined as household contacts, immediate neighbours and next neighbours. The data on patients and the general population data are being further analysed.

On all islands, the leprosy patients were treated with MDT according to the national guidelines. To be able to study two different regimens for prophylactic treatment (blanket treatment and contact treatment) and to have a control group, the three small islands, Pelokan, Kembanglemari and Tampaan, were combined to serve as one group (1252 inhabitants, 39 leprosy patients). The two larger islands, Sapuka (2069 inhabitants, 26 leprosy patients) and Sailus (1449 inhabitants, 26 patients) each served as one group. Sailus served as the controlisland, in which only the patients were treated with MDT. On the group of three small islands, $79 \%$ of the population (persons without leprosy; above 5 years of age and without contraindication) received prophylactic treatment with rifampicin. On Sapuka, contacts of patients (household contacts, immediate neighbours and next neighbours, accounting for $17 \%$ of the population) received prophylactic treatment. During the delivery of the prophylaxis to both groups, $73 \%$ took their medication under supervision, whereas $27 \%$ of those treated self-administered the medication. Prophylaxis will be repeated after 4-5 months.

During screening, blood obtained by venepuncture and nasal-swabs were collected from everyone over 5 yeais of age. Screening and collection of blood-samples and nasal swabs will be repeated annually for 3 years. This will permit us to observe the effects of the different prophylactic regimens on the incidence of leprosy and the transmission of $M$. leprae.

\section{References}

I Visschedijk J, Van de Broek, J, Eggens H et al. Mycobacterium leprae-millennium resistant! Leprosy control on the threshold of a new era. Trop Med Int Health, 2000; 5: 388-399.

2 Van Beers SM, deWit MYL, Klatser PR. The epidemiology of Mycobacterium leprae: recent insight. FEMS Microbiol Lett, 1996; 136: 221-230. 
3 Van Beers SM, Hatta M, Klatser PR. Patient contact is the major determinant in incident leprosy: implications for future control. Int J Lepr, 1999; 67: 119-128.

\section{DISCUSSION}

Dr Gupte: I have a question with respect to the 'stone-in-the-pond' model. This epidemiologic pattern has also been observed in the case of cholera. After the stone falls into the pond, the ripples spread centrifugally; they do not return toward the centre. In the case of leprosy, however, some not-very-well-documented observations suggest that the same individual may become ill with leprosy more than once.

Dr Klatser: I can imagine that, in such instances, it may be very difficult to distinguish between reinfection and relapse.

Dr Gillis: In order to distinguish between reinfection and relapse, there is a need to look for strain-specific markers.

Dr Dockrell: Your work has been carried out in an area of high endemicity. What would the situation be in an area of low endemicity? Dr Gupte has discussed the situation in states like Rajasthan, in which isolated new cases continue to be detected. Is it possibly necessary to have a high background in order to observe the clusters of households and patients that you have described?

Professor Grosset: In tuberculosis, the 'stone' in the pond analogy is the smear-positive patients. However, you have described many cases of leprosy among the household contacts of PB patients. In these cases, there must have been alternative sources of infection. In a situation of high endemicity, no one knows who is the source of infection. Therefore, the importance of strain typing of M. leprae, as is currently done for M. tuberculosis.

Dr Noordeen: I think that extending the circle of contacts to include the neighbourhood is very interesting. Dr Convit developed a method for identifying non-household contacts, but it was not entirely accurate. In addition, it should be recognised that the risk of leprosy among household contacts is not the same everywhere, and varies with the degree of endemicity in the community. 